\title{
ON UNIVERSAL FUNCTIONS
}

\author{
Richard Aron and Dinesh Markose
}

\begin{abstract}
An entire function $f \in \mathcal{H}(\mathbb{C})$ is called universal with respect to translations if for any $g \in \mathcal{H}(\mathbb{C}), R>0$, and $\epsilon>0$, there is $n \in \mathbb{N}$ such that $|f(z+n)-g(z)|<\epsilon$ whenever $|z| \leq \mathrm{R}$. Similarly, it is universal with respect to differentiation if for any $g, R$, and $\epsilon$, there is $n$ such that $\left|f^{(n)}(z)-g(z)\right|<\epsilon$ for $|z| \leq \mathrm{R}$. In this note, we review G. MacLane's proof of the existence of universal functions with respect to differentiation, and we give a simplified proof of $G$. D. Birkhoff's theorem showing the existence of universal functions with respect to translation. We also discuss Godefroy and Shapiro's extension of these results to convolution operators as well as some new, related results and problems.
\end{abstract}

\section{Introduction}

Our interest here will be in what has come to be called hypercyclic operators on the space $\mathcal{H}(\mathbb{C})$ of entire functions of one complex variable. This subject has its origins in 1929 with the paper [2] by G. D. Birkhoff, in which he proved that there is $f \in \mathcal{H}(\mathbb{C})$ such that the set of all translates $\{f(z), f(1+z), \ldots, f(n+z), \ldots\}$ is dense in $\mathcal{H}(\mathbb{C})$. About 25 years later, G. MacLane [9] proved an analogous result for derivatives: There is an entire function $f$ such that the set of all derivatives $\left\{f, f^{\prime}, \ldots, f^{(n)}, \ldots\right\}$ is dense in $\mathcal{H}(\mathbb{C})$. We present an exposition of these results in $\S 2$, presenting a somewhat simpler, and certainly shorter, proof of Birkhoff's result.

Recall that an operator $T: X \rightarrow X$ is said to be hypercyclic if there is some (hypercyclic) vector $x \in X$ such that $\left\{x, T(x), \ldots, T^{n}(x), \ldots\right\}$ is dense in $X$. These two results can be restated in terms of hypercyclic operators on $\mathcal{H}(\mathbb{C})$, by simply noting that Birkhoff's result means that the translation operator $T: \mathcal{H}(\mathbb{C}) \rightarrow \mathcal{H}(\mathbb{C}), T(h)(z) \equiv h(1+z)$ is

Received November 9, 2002.

2000 Mathematics Subject Classification: Primary 47A16, 32DXX; Secondary $46 \mathrm{~B} 37$.

Key words and phrases: hypercyclic, analytic functions, convolution operators. 
hypercyclic. Likewise, MacLane's result just says that the differentiation operator is hypercyclic. This fundamental observation was made by Godefroy and Shapiro [6] who generalized it to show that every continuous linear operator $L: \mathcal{H}(\mathbb{C}) \rightarrow \mathcal{H}(\mathbb{C})$ which commutes with translations and which is not a multiple of the identity is hypercyclic. In $\S 3$, we review their result and the connection with so-called convolution operators. We also present examples of new hypercyclic operators and pose some problems related to these operators.

\section{Classical results of Birkhoff and MacLane}

TheOREM 1. [2] There is a function $f \in \mathcal{H}(\mathbb{C})$ with the following property: For every $g \in \mathcal{H}(\mathbb{C})$ and every $R, \epsilon>0$, there is $n \in \mathbb{N}$ such that $|f(z+n)-g(z)|<\epsilon$ for every $z \in \mathbb{C},|z| \leq R$.

Proof. Let $\left(P_{j}\right)_{j}$ be a dense sequence of polynomials in $\mathcal{H}(\mathbb{C})$. To simplify the argument, let's assume that each $P_{j}$ occurs infinitely often in this sequence. Let $\left(D_{j}\right)_{j}$ be a sequence of disjoint closed discs, each $D_{j}$ of radius $j$, such that the centers $\left(c_{j}\right)$ form an increasing sequence on the positive real axis. Let $E_{j}$ be a sequence of closed discs, each centered at the origin, such that $D_{j} \subset E_{j}$ and $D_{j+1} \cap E_{j}=\emptyset$. (Hence, $D_{i} \subset E_{j}$ for $1 \leq i \leq j$ and $D_{i} \cap E_{j}=\emptyset$ for $i \geq j+1$.)

Call $Q_{1}=P_{1}$. By Runge's theorem, there is a polynomial $Q_{2}$ such that $\left\|Q_{2}\right\|_{E_{1}}<\frac{1}{2}$ and such that

$$
\left|Q_{2}(z)-\left(P_{2}\left(z-c_{2}\right)-Q_{1}(z)\right)\right|<\frac{1}{2}
$$

on $D_{2}$. Next, choose a polynomial $Q_{3}$ such that $\left\|Q_{3}\right\|_{E_{2}}<\frac{1}{2^{2}}$ and such that

$$
\left|Q_{3}(z)-\left(P_{3}\left(z-c_{3}\right)-Q_{1}(z)-Q_{2}(z)\right)\right|<\frac{1}{2^{2}}
$$

on $D_{3}$. In general, let $Q_{n}$ be a polynomial such that $\left\|Q_{n}\right\|_{E_{n-1}}<\frac{1}{2^{n-1}}$ and such that

$$
\left|Q_{n}(z)-\left(P_{n}\left(z-c_{n}\right)-\sum_{i=1}^{n-1} Q_{i}(z)\right)\right|<\frac{1}{2^{n-1}}
$$

on $D_{n}$.

We claim that the function $f=\sum_{n=1}^{\infty} Q_{n}$ works. It is easy to see that $f$ is entire, and so there only remains to show that if $g \in \mathcal{H}(\mathbb{C}), R>0$, and $\epsilon>0$ are arbitrary, then for some $j,\left|f\left(z+c_{j}\right)-g(z)\right|<2 \epsilon$ whenever $|z| \leq R$. In fact, it is enough to demonstrate this for $g=P=P_{k}$, for 
some $k$. Noting that there are infinitely many $k$ for which $P=P_{k}$, we can choose such a large $k$ so that

$$
\left\|f-\sum_{i=1}^{k} Q_{i}\right\|_{E_{k-1}}<\epsilon .
$$

Also,

$$
\left|\sum_{i=1}^{k} Q_{i}(z)-P\left(z-c_{k}\right)\right|<\epsilon
$$

for all $z \in D_{k}$. In other words, for all $z \in D_{k},\left|f(z)-P\left(z-c_{k}\right)\right|<2 \epsilon$, and the result follows by a change of variable.

We next present the original argument of MacLane, which shows that there is an entire function whose collection of derivatives is dense in $\mathcal{H}(\mathbb{C})$. The method of proof will be useful to us later.

TheOREM 2. [9] There is an entire function $f$ such that the set $\left\{f^{n}: n \in \mathbb{N}\right\}$ is dense in $\mathcal{H}(\mathbb{C})$.

Proof. Let $I: \mathcal{H}(\mathbb{C}) \rightarrow \mathcal{H}(\mathbb{C})$ be defined by

$$
I(h)(z)=\int_{0}^{z} h(w) d w .
$$

Note that if $g(z)=z^{n}$, then $I(g)(z)=\frac{z^{n+1}}{n+1}$ and, in general,

$$
I^{k} g(z)=\frac{z^{n+k}}{(n+k) \cdots(n+1)} \text {. }
$$

So, for $|z| \leq R$,

$$
\left|I^{k} g(z)\right| \leq \frac{R^{n+k}}{(n+k) \cdots(n+1)} \leq R^{n} \frac{R^{k}}{k !} .
$$

Thus, $\max _{|z| \leq R}\left|I^{k} g(z)\right| \rightarrow 0$ as $k \rightarrow \infty$, and from this it is obvious that given any polynomial $P$, any $\delta>0$ and any $R>0$, there is $\tilde{k} \in \mathbb{N}$ such that $\max _{|z| \leq R}\left|I^{k} P(z)\right|<\delta$ whenever $k \geq \tilde{k}$. Moreover, given $h \in \mathcal{H}(\mathbb{C})$, $\epsilon>0$, and $M \in \mathbb{N}$, if $|h(z)| \leq \delta$ whenever $|z| \leq R$, then

$$
\begin{aligned}
\left|h^{(j)}(w)\right| & \leq \frac{j ! \max _{|z| \leq R}|h(z)|}{(R / 2)^{j}} \text { for any }|w| \leq R / 2, \\
& \leq j !(2 / R)^{j} \delta<\epsilon
\end{aligned}
$$

for any $j=0, \ldots, M$, provided $\delta$ is small enough. 
To summarize, given any polynomial $P$, any $R, \epsilon>0$, and any $M \in$ $\mathbb{N}$, there is some $\tilde{k} \in \mathbb{N}$ such that if $k \geq \tilde{k}$ and $Q(z) \equiv I^{k} P(z)$, then

$$
\max _{|z| \leq R}\left|Q^{(j)}(z)\right|<\epsilon
$$

for every $j=0, \ldots, M$.

With this, we are now ready to prove the theorem. Let $\left\{P_{j}: j \in \mathbb{N}\right\}$ be a dense sequence of polynomials in $\mathcal{H}(\mathbb{C})$. The required function $f$ will have the form

$$
f=\sum_{j=1}^{\infty} I^{k_{j}}\left(P_{j}\right) .
$$

Let $k_{1}=0, Q_{1}=P_{1}$, and choose $k_{2}>k_{1}+\operatorname{deg} P_{1}$. Call $Q_{2}=I^{k_{2}}\left(P_{2}\right)$, where $k_{2}$ is chosen to further satisfy the condition that $\left|Q_{2}(z)\right|<\frac{1}{2^{2}}$ for $|z| \leq 2$. Next, let $k_{3}>k_{2}+\operatorname{deg} P_{2}$, and call $Q_{3}=I^{k_{3}}\left(P_{3}\right)$ where $k_{3}$ has been chosen to further satisfy the conditions that

$$
\left|Q_{3}(z)\right| \leq \frac{1}{2^{3}},\left|Q_{3}^{\prime}(z)\right| \leq \frac{1}{2^{3}}, \ldots,\left|Q_{3}^{\left(k_{2}\right)}(z)\right| \leq \frac{1}{2^{3}}
$$

on $|z| \leq 3$. In general, let $k_{n}>k_{n-1}+\operatorname{deg} P_{n-1}, k_{n}$ so large that if $Q_{n}=I^{\overline{k_{n}}}\left(P_{n}\right)$, then

$$
\left|Q_{n}(z)\right| \leq \frac{1}{2^{n}},\left|Q_{n}^{\prime}(z)\right| \leq \frac{1}{2^{n}}, \ldots,\left|Q_{n}^{\left(k_{n-1}\right)}(z)\right| \leq \frac{1}{2^{n}}
$$

on $|z| \leq n$. Let $f=\sum_{n=1}^{\infty} Q_{n}$. We claim that $f$ works. First, since $\max _{|z| \leq n}\left|Q_{n}(z)\right| \leq \frac{1}{2^{n}}$, it is clear that the series converges to $f$ uniformly on bounded subsets of $\mathbb{C}$. Second, let $g \in \mathcal{H}(\mathbb{C}), R>0$, and $\epsilon>0$ be arbitrary. We choose $n_{0} \in \mathbb{N}$ so that $n_{0}>R$ and $\frac{1}{2^{n_{0}-1}}<\epsilon$. Choose $n \geq$ $n_{0}$ such that $\max _{|z| \leq n_{0}}\left|g(z)-P_{n}(z)\right|<\epsilon$. We claim that $\max _{|z| \leq n_{0}} \mid g(z)-$ $f^{\left(k_{n}\right)}(z) \mid<2 \epsilon$. In fact, this is very simple since if $|z| \leq n$, then

$$
\left|g(z)-f^{\left(k_{n}\right)}(z)\right| \leq\left|g(z)-P_{n}(z)\right|+\sum_{j>n}\left|Q_{j}^{\left(k_{n}\right)}\right|<2 \epsilon .
$$

C. Blair and L. Rubel [3] were perhaps the first to observe that one can find a function $f \in \mathcal{H}(\mathbb{C})$ satisfying the conclusions of both Theorem 1 and Theorem 2. Their proof involves a combination of the arguments given above, together with a slightly more complicated application of Runge's theorem. As we will see in the next section, the Blair-Rubel result is subsumed by several other considerably stronger theorems. 


\section{Convolution operators}

The basis for this section is the very beautiful paper [6] of G. Godefroy and J. Shapiro, which in turn relies heavily on the independent work of C. Kitai [8] and R. Gethner and J. Shapiro [5]. These papers provide a sufficient condition for the hypercyclicity of operators on Fréchet spaces. We recall their hypercyclicity condition in Theorem 5, below. First, however, we review some notions which will be fundamental for the remainder of this note.

Let $\mathcal{O}(\mathcal{H}(\mathbb{C}))=\{L: \mathcal{H}(\mathbb{C}) \rightarrow \mathcal{H}(\mathbb{C}): L$ is continuous, linear, and commutes with translations $\}$. In other words, $\mathcal{O}(\mathcal{H}(\mathbb{C}))$ consists of all linear, continuous operators $L$ such that for any $a \in \mathbb{C}$ and $f \in \mathcal{H}(\mathbb{C})$, we have $L\left(\tau_{a} f\right)=\tau_{a}(L f)$; here $\tau_{a} f(z)=f(z+a)$. The elements of $\mathcal{O}(\mathcal{H}(\mathbb{C}))$ are called convolution operators. The easiest non-trivial examples of such operators are those described in Theorems 1 and 2 above, namely $L(f)(z)=f(z+a)$ and $L(f)=f^{\prime}$. In what follows, entire functions of exponential type will play a major role. Recall that $\operatorname{Exp}(\mathbb{C})=\{g \in$ $\mathcal{H}(\mathbb{C})$ : for some $C, R>0,|g(z)| \leq C e^{R|z|}$ for every $\left.z \in \mathbb{C}\right\}$.

Proposition 3. The spaces $\mathcal{O}(\mathcal{H}(\mathbb{C})), \mathcal{H}^{\prime}(\mathbb{C})$ (the dual of $\mathcal{H}(\mathbb{C})$ ), and $\operatorname{Exp}(\mathbb{C})$ are isomorphic as vector spaces.

Sketch of Proof. (1) Given $L \in \mathcal{O}\left(\mathcal{H}(\mathbb{C})\right.$ ), define $T_{L}: \mathcal{H}(\mathbb{C}) \rightarrow \mathbb{C}$ by $T_{L}(f)=L(f)(0)$. Conversely, given $T \in \mathcal{H}^{\prime}(\mathbb{C})$, let $L_{T}: \mathcal{H}(\mathbb{C}) \rightarrow \mathcal{H}(\mathbb{C})$ be given by $L_{T}(f)(w)=T\left(\tau_{w} f\right)$. It is routine that $L_{T_{L}}=L$ for every $L \in \mathcal{O}(\mathcal{H}(\mathbb{C}))$, and conversely that $T_{L_{T}}=T$ for every $T \in \mathcal{H}^{\prime}(\mathbb{C})$.

(2) Given $T \in \mathcal{H}^{\prime}(\mathbb{C})$, let $\hat{T}: \mathbb{C} \rightarrow \mathbb{C}$ be given by $\hat{T}(\lambda)=T\left(e^{\lambda z}\right)$. Since $T$ is continuous, there are $C$ and $R>0$ such that $|T(h)| \leq$ $C \max _{|z| \leq R}|h(z)|$. Therefore $|\hat{T}(\lambda)| \leq C \max _{|z| \leq R}\left|e^{\lambda z}\right|=C e^{R|\lambda|}$. For the other direction, given an entire function $g$ which is of exponential type, let $T_{g} \in \mathcal{H}^{\prime}(\mathbb{C})$ be defined by $T_{g}(f)=\sum_{n=0}^{\infty} \frac{g^{(n)}(0)}{n !} f^{(n)}(0)$. Since an entire function $g$ is in $\operatorname{Exp}(\mathbb{C})$ if and only if $\sup _{n}\left|g^{(n)}(0)\right|^{\frac{1}{n}}<\infty$, the convergence of $T_{g}$ easily follows.

As an example, the convolution operator given by translation, $L(f)(z)$ $=f(z+1)$, corresponds to the linear form $T_{L}(f)=f(1)$, which in turn corresponds to the entire function $e^{\lambda}$ of exponential type. Note that $f(z+1)=\sum_{n=0}^{\infty} \frac{f^{(n)}(z)}{n !}$, which explains why convolution operators are sometimes called infinite order differential operators. 
We next indicate why the three spaces are isomorphic as algebras. At first glance, this may seem surprising: $\operatorname{Exp}(\mathbb{C})$ is obviously a commutative algebra under pointwise multiplication. On the other hand, while $\mathcal{O}(\mathcal{H}(\mathbb{C}))$ is an algebra under composition of operators, it is not at all clear that it is commutative. Finally, there is no obvious multiplication on $\mathcal{H}^{\prime}(\mathbb{C})$. To remedy this, for $S$ and $T \in \mathcal{H}^{\prime}(\mathbb{C})$, let $S * T: \mathcal{H}(\mathbb{C}) \rightarrow \mathbb{C}$ be defined by $S * T(f)=S(T * f)$ where, in turn, $T * f(\lambda)=T\left(\tau_{\lambda} f\right)$. It is not difficult to verify that for $L_{1}, L_{2} \in \mathcal{O}(\mathcal{H}(\mathbb{C})), T_{L_{1} \circ L_{2}}=T_{L_{1}} * T_{L_{2}}$, and furthermore for $T_{1}, T_{2} \in \mathcal{H}^{\prime}(\mathbb{C}), \widehat{T_{1} * T_{2}}=\hat{T}_{1} \hat{T}_{2}$. The conclusion is summarized in the following:

Proposition 4. The three spaces $\mathcal{O}(\mathcal{H}(\mathbb{C})), \mathcal{H}^{\prime}(\mathbb{C})$, and $\operatorname{Exp}(\mathbb{C})$ are isomorphic as algebras.

We omit the proof of the following fundamental theorem, due independently to C. Kitai and R. Gethner and J. Shapiro. The proof makes use of only the Baire category theorem (plus some cleverness).

Theorem 5. (Hypercyclicity Condition) $[5,8]$. Let $T: X \rightarrow X$ be a continuous linear operator on a Fréchet space $X$. Suppose that $Y$ and $Z$ are dense subsets of $X$ and that there is a mapping $S: Z \rightarrow Z$ such that the following three conditions are satisfied:

(i) for all $y \in Y, T^{n}(y) \rightarrow 0$ as $n \rightarrow \infty$,

(ii) for all $z \in Z, S^{n}(z) \rightarrow 0$ as $n \rightarrow \infty$,

(iii) $T \circ S=I_{Z}$.

Then $T$ is hypercyclic; that is, there is a vector $x_{0} \in X$ such that the set $\left\{x_{0}, T x_{0}, \ldots, T^{n} x_{0}, \ldots\right\}$ is dense in $X$.

Note that in the above, $Y$ and $Z$ need not be vector spaces, nor is it necessary that $S$ be linear or continuous.

The following result was originally proved for convolution operators acting on $\mathcal{H}\left(\mathbb{C}^{n}\right)$. In order to maintain a consistent presentation, we have opted to state and prove it only in the one variable case (although there are no particular difficulties in extending the argument to $\mathcal{H}\left(\mathbb{C}^{n}\right)$ ).

Theorem 6. [6] Let $L \in \mathcal{O}(\mathcal{H}(\mathbb{C}))$ be a convolution operator which is not a multiple of the identity. Then $L$ is hypercyclic.

Proof of Theorem. Let $L \in \mathcal{O}(\mathcal{H}(\mathbb{C}))$ be any convolution operator which is not a multiple of the identity. By Proposition 3 , corresponding to $L$ is a unique function $g_{L} \in \operatorname{Exp}(\mathbb{C})$, where if $g_{L}(z)=\sum_{j=0}^{\infty} a_{j} z^{j}$ then $L(f)(z)=\sum_{j=0}^{\infty} a_{j} f^{(j)}(z) \quad(z \in \mathbb{C}, f \in \mathcal{H}(\mathbb{C}))$. It is useful to observe that $L$ is a multiple of the identity if and only if the associated function $g_{L}$ is constant. Note also that for each fixed $b \in \mathbb{C}$, the function $f_{b}(z)=e^{b z}$ is 
an eigenvector of $L: L\left(f_{b}\right)(z)=\sum_{j} a_{j} b^{j} f_{b}(z)=g_{L}(b) f_{b}(z)$. To continue the proof, we will need the following result:

Sublemma 7. For each open, non-empty subset $V \subset \mathbb{C}, \operatorname{span}\left\{f_{b}\right.$ : $b \in V\}$ is dense in $\mathcal{H}(\mathbb{C})$.

Proof of Sublemma. Fix $b \in V$. For all sufficiently small $\delta>0, b+\delta \in$ $V$ and it is easy to verify that

$$
\lim _{\delta \rightarrow 0} \frac{e^{(b+\delta) z}-e^{b z}}{\delta} \rightarrow z e^{b z}
$$

in $\mathcal{H}(\mathbb{C})$. Thus, $z e^{b z} \in \overline{\operatorname{span}\left\{f_{b}: b \in V\right\}}$. Next, $z^{2} e^{b z} \in \overline{\operatorname{span}\left\{f_{b}: b \in V\right\}}$ since $\frac{e^{b z}\left[e^{\delta z}-1-\delta z\right]}{\delta} \in \overline{\operatorname{span}\left\{f_{b}: b \in V\right\}}$. Continuing, we conclude that for every $n \in \mathbb{N}, z^{n} e^{b z} \in \overline{\operatorname{span}\left\{f_{b}: b \in V\right\}}$. Now, let $f \in \mathcal{H}(\mathbb{C})$ be arbitrary, and let $\sum_{n=0}^{\infty} a_{n} z^{n}$ be the Taylor series of $g(z)=e^{-b z} f(z)$. Thus, $f$ can be represented as $f(z)=e^{b z} g(z)=\sum_{n=0}^{\infty} a_{n} z^{n} e^{b z}$, and the Sublemma is proved.

Returning to the proof of Theorem 6 , let $V=\left\{b \in \mathbb{C}:\left|g_{L}(b)\right|<1\right\}$ and let $W=\left\{b \in \mathbb{C}:\left|g_{L}(b)\right|>1\right\}$. Note that both $V$ and $W$ are open and non-empty, since $g_{L}$ is non-constant. Let's apply the hypercyclicity condition with $Y=\operatorname{span}\left\{f_{b}: b \in V\right\}$ and $Z=\operatorname{span}\left\{f_{b}: b \in W\right\}$, and verify conditions (i), (ii), and (iii) of Theorem 5 . First, (i) follows directly from the fact that $L^{n}\left(f_{b}\right)=g_{L}(b)^{n} f_{b} \rightarrow 0$ as $n \rightarrow \infty$ for each $b \in V$. As for (ii) and (iii), define $S: Z \rightarrow Z$ by $S\left(f_{b}\right)(z)=\frac{f_{b}(z)}{g_{L}(b)}$ for $b \in W$, extending linearly to all of $Z$. Note that $S$ is well-defined and clearly satisfies $L \circ S\left(f_{b}\right)=f_{b}$ for all $b \in W$. Thus (iii) holds. As for (ii), since $\left|g_{L}(b)\right|>1$, it is clear that $S^{n}\left(f_{b}\right) \rightarrow 0$ for each $b \in W$, and hence $S^{n} \rightarrow 0$ pointwise on $Z$.

The proof of the Sublemma is somewhat more direct than that given in [6]. By this Sublemma, $\operatorname{span}\left\{f_{b}: b \in \mathbb{C}\right\}$ is dense in $\mathcal{H}(\mathbb{C})$. Since each $f_{b}$ is in the range of $L$, the following is straightforward:

COROLLARY 8. Every non-zero convolution operator $L$ has dense range.

Proof. Let $L \neq 0$ be a convolution operator. If $L=c I$, then it is clear that $L$ is surjective. Otherwise, Sublemma 7 and the proof of Theorem 6 above apply, to give that each $f_{b} \in$ range $L$, and therefore $\mathcal{H}(\mathbb{C})=\overline{\operatorname{span}\left\{f_{b}: b \in \mathbb{C}\right\}}=\overline{L(\mathcal{H}(\mathbb{C}))}$

At this point, we should mention that much more is true. In fact, it is a result of Malgrange [10] that every non-zero convolution operator $L$ 
is surjective. Since we already have most of the preliminaries, we will sketch this argument in Theorem 11, which was extended by C. Gupta [7] to the class of nuclear entire functions of bounded type on a Banach space. In fact, Gupta also characterized the kernel of an arbitrary convolution operator. The proof rests on the following technical lemma:

Lemma 9. [10] Suppose that $g_{1}, g_{2}$, and $g_{3}$ are in $\mathcal{H}(\mathbb{C})$, such that $g_{1}=g_{2} \cdot g_{3}$ and $g_{2} \neq 0$. If both $g_{1}$ and $g_{2}$ are in $\operatorname{Exp}(\mathbb{C})$, then so is $g_{3}$.

Proposition 10. Suppose that $T_{1}$ and $T_{2}$ are in $\mathcal{H}^{\prime}(\mathbb{C})$ with $T_{2} \neq 0$. Suppose that for all complex polynomials $P$ and all $\lambda \in \mathbb{C}$, the following implication holds: If $T_{2} *\left(P(z) e^{\lambda z}\right)=0$, then $T_{1}\left(P(z) e^{\lambda z}\right)=0$. Then

$$
\frac{\hat{T}_{1}}{\hat{T}_{2}} \in \operatorname{Exp}(\mathbb{C}) \text {. }
$$

Proof. Suppose that $\lambda$ is a zero of order $k$ of $\hat{T}_{2}$. Then $\hat{T}_{2}^{(j)}(\lambda)=0$ for every $j<k$, and a computation shows that then $T_{2}\left(z^{j} e^{\lambda z}\right)=0$ for all such $j$. By hypothesis, $T_{1}\left(z^{j} e^{\lambda z}\right)=0$ as well, which implies that $\lambda$ is a zero of (at least) order $k$ of $\hat{T}_{1}$. This proves that $\frac{\hat{T}_{1}}{\hat{T}_{2}} \in \mathcal{H}(\mathbb{C})$ and the conclusion follows from Proposition 3 and Lemma 9.

ThEOREM 11. [10] If $L \in \mathcal{O}(\mathcal{H}(\mathbb{C}))$ is non-zero, then it is surjective.

Proof. The proof makes use of the Dieudonné-Schwartz theorem [4]: If $u: E \rightarrow F$ is a linear continuous operator between Fréchet spaces $E$ and $F$, then $u$ is surjective if and only if $u^{t}$ is injective and $u^{t}$ has weakstar closed range in $E^{\prime}$. So, in order to prove the theorem, it suffices to show that $L^{t}$ has weak-star closed range and is injective. As for the latter assertion, suppose that $L^{t}(S)=0$ for some $S \in \mathcal{H}^{\prime}(\mathbb{C})$. Therefore, for all $f \in \mathcal{H}(\mathbb{C}), L^{t} S(f)=S(L f)=S\left(T_{L} * f\right)=S * T_{L}(f)=0$. Consequently, $S * T_{L}=0$, and so $\hat{S} \cdot \hat{T}_{L}=0$. Since $L \neq 0$, it follows that $\hat{T}_{L} \neq 0$. Thus, $\hat{S}=0$, and so $S=0$.

Next, let's show that $L^{t}$ has weak-star closed range. In fact, we'll show more, namely that

$$
\text { (*) } \quad L^{t}\left(\mathcal{H}^{\prime}(\mathbb{C})\right)=\{f \in \mathcal{H}(\mathbb{C}): L(f)=0\}^{\perp} .
$$

This equality will prove the result, since $\{f \in \mathcal{H}(\mathbb{C}): L(f)=0\}^{\perp}=$ $\cap\left\{T \in \mathcal{H}^{\prime}(\mathbb{C}): T(f)=0\right\}$, the intersection being over all $f$ such that $L(f)=0$. Now, each set $\left\{T \in \mathcal{H}^{\prime}(\mathbb{C}): T(f)=0\right\}$ is clearly weak-star closed, and it follows that $L^{t}\left(\mathcal{H}^{\prime}(\mathbb{C})\right)$ is weak-star closed. To prove $\left(^{*}\right)$ above, take a functional of the form $L^{t}(T)$. For any $f$ such that $L(f)=0$, $L^{t}(T)(f)=T(L(f))=0$, and so we have proved that the left hand side 
of $\left(^{*}\right)$ is contained in the right hand side. For the converse, suppose that $T \in\{f \in \mathcal{H}(\mathbb{C}): L(f)=0\}^{\perp}$. By Proposition 10,

$$
\frac{\hat{T}}{\hat{L}} \in \operatorname{Exp}(\mathbb{C})
$$

Proposition 4 yields that $\hat{T}=\hat{S} \cdot \hat{L}$ for some $S \in \mathcal{H}^{\prime}(\mathbb{C})$, and consequently $T=S * L$. Thus, for any entire function $f, T(f)=S * L(f)=$ $S(L(f))=L^{t}(S)(f)$. In other words, $T$ belongs to $L^{t}\left(\mathcal{H}^{\prime}(\mathbb{C})\right)$, and the proof is finished.

Let us now return to hypercyclicity, by mentioning another consequence of Theorem 6.

Corollary 12. Given a convolution operator $L$ which is not a multiple of the identity, there is a dense vector space $M \subset \mathcal{H}(\mathbb{C})$ such that for every $f \in M, f \neq 0$, the set $\left\{f, L(f), \ldots, L^{n}(f), \ldots\right\}$ is dense in $\mathcal{H}(\mathbb{C})$. In other words, there is a dense subspace of $\mathcal{H}(\mathbb{C})$, every non-zero element of which is hypercyclic for $L$.

Proof. Let $M=\{P(L)(f): P$ is a polynomial $\}$, where $f$ is hypercyclic for $L$. By Corollary 8 , for any $P \neq 0, P(L): \mathcal{H}(\mathbb{C}) \rightarrow \mathcal{H}(\mathbb{C})$ has dense range. Thus, for each non-zero $P,\left\{L^{n}(P(L)(f)): n \in\right.$ $\mathbb{N} \cup\{0\}\}=P(L)\left(\left\{L^{n}(f): n \in \mathbb{N}\right\}\right)$ is dense in $\mathcal{H}(\mathbb{C})$. In other words, every non-zero element of $M$ is hypercyclic for $L$.

We conclude with some recent partial results and some open problems. First, we do not know of a completely satisfactory extension of Theorem 6 to convolution operators acting on a space of entire functions of infinitely many variables. There has been some limited progress in this direction by the first author and J. Bès [1], as well some interesting related work by $\mathrm{H}$. Petersson [11], but the following problem remains open:

Problem. Let $L: \mathcal{H}_{N b}(E) \rightarrow \mathcal{H}_{N b}(E)$ be a convolution operator acting on the space of entire functions of nuclear-bounded type on a Banach space $E$ having separable dual. (See [7] for background notation and terminology.) If $L$ is not a multiple of the identity map, is $L$ hypercyclic?

Second, and perhaps of greater interest, is the question of what other operators $L: \mathcal{H}(\mathbb{C}) \rightarrow \mathcal{H}(\mathbb{C})$ are hypercyclic? We show here that certain analogues of the differentiation operator are hypercyclic, while others are not. 
Theorem 13. For $\lambda, b \in \mathbb{C}$, define $T: \mathcal{H}(\mathbb{C}) \rightarrow \mathcal{H}(\mathbb{C})$ by $T(f)(z)=$ $f^{\prime}(\lambda z+b)$. Then $T$ is hypercyclic provided $|\lambda| \geq 1$.

Proof. Since the case when $\lambda=1$ has already been covered in Theorems 2 and 6 , there is no loss in generality in assuming that $\lambda \neq 1$. The proof consists in applying the Hypercyclicity Condition of Theorem 5 , where $Y=Z$ will be the space of analytic polynomials in $X=\mathcal{H}(\mathbb{C})$. A straightforward calculation shows that

$$
T^{n} f(z)=\lambda^{\frac{n(n-1)}{2}} f^{(n)}\left(\lambda^{n} z+\left[\frac{1-\lambda^{n}}{1-\lambda}\right] b\right) .
$$

So, condition (i) of Theorem 5 is satisfied. (In fact, it is satisfied for any $\lambda \in \mathbb{C}$.) Next, let $S: Z \rightarrow Z$ be defined as follows:

$$
S\left(z^{k}\right) \equiv \frac{1}{k+1}\left(\frac{z-b}{\lambda}\right)^{k+1}
$$

and extended linearly. It is easy to verify that $T \circ S\left(z^{k}\right)=z^{k}$, so that condition (iii) of Theorem 5 is also true. As for condition (ii),

$$
S^{n}\left(z^{k}\right)=\frac{k !}{(k+n) !}\left(\frac{z-b[1+\lambda]}{\lambda^{n}}\right)^{k+n} .
$$

Therefore, for any $R>0$,

$$
\max _{|z| \leq R}\left|S^{n}\left(z^{k}\right)\right| \leq \frac{k !}{(k+n) !}(R+|b|(1+|\lambda|))^{k+n},
$$

since $|\lambda| \geq 1$, which tends to 0 as $n \rightarrow \infty$. Thus, condition (ii) holds for all $z^{k}$ and therefore it holds for all polynomials.

It should be noted that unless $\lambda=1$, the operator $T$ is not a convolution operator; although it is clearly linear and continuous, $T$ does not commute with translations. One particular case of the above result is noteworthy. If we let $T(f)(z)=f^{\prime}\left(e^{2 \pi i \frac{p}{q}} z\right)$ where $p, q \in \mathbb{N}$, then $T$ is hypercyclic. A direct argument for this result is easy, since $T^{q}(f)=f^{(q)}$ which is a convolution operator. It is trivial that if a power of an operator is hypercyclic then the operator itself is hypercyclic, and so $T$ is seen to be hypercyclic. However, there does not seem to be a way to use this easy observation to conclude that $T(f)(z)=f^{\prime}(\lambda z)$ is hypercyclic if $|\lambda|=1, \lambda \neq e^{2 \pi i \frac{p}{q}}$.

The following result complements the preceeding Theorem by showing that in a sense, the "weighted differentiation" operator $T_{\lambda}, T_{\lambda}(f)(z)$ $=f^{\prime}(\lambda z)$, acting on $\mathcal{H}(\mathbb{C})$ behaves in an analogous manner to the weighted backward shift $B_{\lambda}: \ell_{2} \rightarrow \ell_{2}$. In the latter case, $B_{\lambda}$ is hypercyclic if 
and only if $|\lambda|>1$. Indeed, the condition is obviously necessary since if $|\lambda| \leq 1$, then $\left\|B_{\lambda}\right\| \leq 1$. In Theorem 13, we showed that $T_{\lambda}$ is hypercyclic if $|\lambda| \geq 1$, and we now show that this condition on $\lambda$ is also necessary.

Proposition 14. For any $\lambda \in \mathbb{C}$ such that $|\lambda|<1$ and any $f \in$ $\mathcal{H}(\mathbb{C})$, the sequence $\left(T_{\lambda}^{n}(f)\right) \rightarrow 0$. Consequently, for these $\lambda, T_{\lambda}$ is not hypercyclic.

Proof. Fix $f$ and $\lambda$ as in the proposition, and let $R>0$. As noted in the proof of the previous theorem,

$$
T_{\lambda}^{n}(f)(z)=\lambda^{\frac{n(n-1)}{2}} f^{(n)}\left(\lambda^{n} z\right) .
$$

Therefore, $\max _{|z| \leq R}\left|T_{\lambda}^{n}(f)(z)\right|=\left|\lambda^{\frac{n(n-1)}{2}}\right| \max _{|z| \leq R}\left|f^{(n)}\left(\lambda^{n} z\right)\right|$. For all large $n$ such that $\left|\lambda^{n} R\right| \leq \frac{1}{2}$ an application of Cauchy's inequality shows that the above is $\leq n !\left|\lambda^{\frac{n(n-1)}{2}}\right| 2^{n} \max _{|w| \leq 1}|f(w)|$. Now by Stirling's formula, this is dominated by an expression of the form

$$
C\left(\frac{n}{e}\right)^{n} 2^{n}\left|\lambda^{\frac{n(n-1)}{2}}\right|=C\left[\frac{2 n|\lambda|^{\frac{n-1}{2}}}{e}\right]^{n} .
$$

Since $n|\lambda|^{\frac{n-1}{2}} \rightarrow 0$, we conclude that $\max _{|z| \leq R}\left|T_{\lambda}^{n}(f)(z)\right| \rightarrow 0$ as $n \rightarrow$ $\infty$.

ACKNOWLEDGEMENT. The authors express their thanks to the Centro de Matematica da Universidade de Coimbra, for its sponsorship of the Special topics in Complex Analysis Program during March, 2002. In addition, the first author expresses his appreciation to friends and colleagues at the Universidad de Valencia, Spain, for their support while a first version of this article was written. Also, the second author expresses his appreciation to friends and colleagues at DPMMS, and especially to Keith Carne, Alan Beardon, Ed Crane and Ian Short for several helpful conversations, suggestions and a lot of sup port. The second author also thanks Trinity College for partially supporting his expenses to attend the Complex Analysis program at Coimbra.

Added in Proof. The authors are grateful to Professor Karl-Goswin Grosse-Erdmann for informing them of the article On universal functions by Wolfgang Luh, which appeared in Fourier analysis and approximation theory (Proc. Colloq., Budapest, 1976), Vol. II, pp. 503-511, Colloq. Math. Soc. János Bolyai, 19, North-Holland, Amsterdam-New York, 1978. Theorem 2 of this paper provides an extremely similar proof of a somewhat stronger version of Birkhoff's Theorem to the one given here. 


\title{
References
}

[1] R. M. Aron and J. Bès, Hypercyclic differentiation operators, in Function spaces (Edwardsville, IL, 1998), 39-46, Contemp. Math., 232, Amer. Math. Soc., Providence, RI, 1999.

[2] G. Birkhoff, Démonstration d'un théoréme elémentaire sur les fonctions entiéres, C. R. Acad. Sci. Paris 189 (1929), 473--475.

[3] C. Blair and L. Rubel, A triply universal entire function, Enseign. Math. (2) 30 (1984), no. 3-4, 269-274.

[4] J. Dieudonné and L. Schwartz, La dualité dans les espaces $(\mathcal{F})$ et $(\mathcal{L F})$, Ann. Inst. Fourier (Grenoble) I (1949), 61-101.

[5] R. M. Gethner and J. H. Shapiro, Universal vectors for operators on spaces of holomorphic functions, Proc. Amer. Math. Soc. 100 (1987), no. 2, 281-288.

[6] G. Godefroy and J. Shapiro, Operators with dense, invariant, cyclic vector manifolds, J. Funct. Anal. 98 (1991), no. 2, 229-269.

[7] C. Gupta, On the Malgrange theorem for nuclearly entire functions of bounded type on a Banach space, Nederl. Akad. Wetensch. Proc. Ser. A73, Indag. Math. 32 (1970), 356-358.

[8] C. Kitai, Invariant closed sets for linear operators, Dissertation, Univ. Toronto, 1982.

[9] G. MacLane, Sequences of derivatives and normal families, J. Anal. Math. 2 (1952), 72-87.

[10] B. Malgrange, Existence et approximation des solutions des équations aux dérivées partielles et des équations des convolution, Ann. Inst. Fourier (Grenoble) VI $(1955 / 56), 271-355$.

[11] Henrik Petersson, Hypercyclic convolution operators on entire functions of Hilbert-Schmidt holomorphy type, Ann. Math. Blaise Pascal 8 (2001), no. 2, 107114.

\author{
Department of Mathematical Sciences \\ Kent State University \\ Kent, Ohio 44242, USA \\ E-mail: aron@mcs.kent.edu \\ Department of Pure Mathematics and Mathematical Statistics \\ Wilberforce Road \\ Cambridge University \\ Cambridge CB3 0WB, UK \\ E-mail: dm295@cam.ac.uk
}

